\title{
Outbreak of Ochrobactrum anthropi endophthalmitis following cataract surgery
}

Fellipe B Mattos ${ }^{1}$; Fábio P Saraiva ${ }^{2}$; Hélio A Neto ${ }^{3}$, Angelo $\mathrm{F} \mathrm{Passos}{ }^{4}$

${ }^{1}$ Physician, Department of Ophthalmology, Federal University of Espírito Santo School of Medicine - UFES - Vitória (ES), Brazil.

${ }^{2}$ Professor and Physician, Department of Ophthalmology, Federal University of Espírito Santo School of Medicine - UFES - Vitória (ES), Brazil.

${ }^{3}$ Physician, Department of Ophthalmology, Federal University of Espírito Santo School of Medicine - UFES - Vitória (ES), Brazil.

${ }^{4}$ Professor and Chair, Department of Ophthalmology, Federal University of Espírito Santo School of Medicine - UFES - Vitória (ES), Brazil.

Department of Ophthalmology, Hospital das Clínicas (Hospital Universitário Cassiano Antônio de Moraes - HUCAM), Federal University of Espírito Santo School of Medicine, Vitória, Espírito Santo, Brazil.

FINANCIAL DISCLOSURE: No author has a financial or proprietary interest in any material or method mentioned.

Corresponding Author: Fellipe Berno Mattos, MD.

Hospital das Clínicas (Hospital Universitário Cassiano Antônio de Moraes) of Federal University of Espírito Santo School of Medicine.

Av. Marechal Campos s/n - Maruípe - Vitória (ES) - Brasil.

CEP 05409-011

Phone: 55 - (27) 33357303; FAX: 55 - (27) 33357100

fellipeberno@yahoo.com.br 


\section{ABSTRACT}

Purpose: To report an investigation of an outbreak of Ochrobactrum anthropi endophthalmitis following cataract surgery and propose a new sterilization protocol to minimize the risk of further cases in the operating environment.

Setting: Hospital das Clínicas, Federal University of Espírito Santo School of Medicine, Vitória, Espírito Santo, Brazil.

Design: Noncomparative consecutive case series.

Methods: The medical records of patients with culture proven $O$. anthropi endophthalmitis or with suggestive clinical findings during the outbreak, but without microbiological confirmation, were reviewed. We also surveyed existing protocols for disinfection and sterilization of surgical materials and discussed their proper application in the hospital routine.

Results: Seven cases of $O$. anthropi pseudophakic endophthalmitis were confirmed between July 24 and November 10, 2010. The average interval between surgery and diagnosis was 5 days. Initial therapy with intravitreal injection of vancomycin and amikacin proved ineffective in 5 of 7 cases (71\%). Complications included macular hole, fibrosis in the macula, retinal vein branch occlusion, retinal ischemia and diffuse hemorrhage, retinal necrosis and phthisis bulbi. Final visual acuity was counting fingers or worse in five patients.

Conclusions: Pars plana vitrectomy with partial capsulectomy appeared to be curative in 2 of 3 surgical cases that could be followed. The most likely cause of this outbreak was contamination of the tubing kit of the phacoemulsification machine. Following introduction of a new sterilization protocol involving the use of an enzymatic detergent and more vigorous cleaning of the tubing with 
distilled water, no further cases were reported in over a thousand subsequent procedures.

KEYWORDS Ochrobactrum anthropi; cataract extraction; endophthalmitis; antibacterial agents; biofilms; sterilization. 


\section{PURPOSE}

Endophthalmitis is a potentially severe intraocular inflammation which may occur as a complication of intraocular surgery or as a result of nonsurgical trauma or systemic infection. Irreversible photoreceptor cell damage is a frequent occurrence, even with prompt and proper therapeutic intervention. ${ }^{1}$

Postoperative endophthalmitis occurs most frequently following cataract surgery, with a reported incidence ranging from 0.02 to $0.3 \% .^{1-8}$

Depending on the time of onset, endophthalmitis may be classified as acute or chronic. The former occurs early (hours or days after surgery) and is caused by virulent organisms. It is often associated with hypopyon and severe inflammation with a commonly aggressive clinical course..$^{8-10}$ The latter is an unusual complication of cataract surgery but well documented in several small series. It is caused by less virulent organisms and the clinical condition is a recurrent, often low-grade uveitis which partially responds to topical or systemic steroids. $^{8-10}$ The indolent course of chronic endophthalmitis is sometimes difficult to distinguish from a persistent postoperative inflammation. It may develop months or even years after the initial surgical event and commonly presents itself with mild pain, granulomatous uveitis with or without hypopyon, vitreous activity, capsular thickening or plaque and visual loss. ${ }^{8-10}$

In this study, we report the clinical aspects of an endophthalmitis outbreak following cataract surgery secondary to Ochrobactrum anthropi at Hospital das Clínicas in Vitória (a state capital of Southeastern Brazil). Our cases differ from the few previously reported cases in many aspects. Surgical management, antibiotic sensitivity and visual outcome are discussed. The 
literature was reviewed in order to develop a sterilization protocol capable of minimizing the risk of further outbreaks in the operating environment.

\section{MATERIALS AND METHODS}

This is an observational case series study. All patients presented with endophthalmitis following cataract surgery with intraocular lens implantation

(IOL) between July 24 and November 10 at Hospital das Clínicas (Vitória, Brazil). The medical records of seven patients with culture-proven $O$. anthropi endophthalmitis or suspected cases without microbiological confirmation observed during the outbreak were reviewed and the clinical presentation of these patients was compared to the few reports found in the literature. We also surveyed existing protocols for disinfection and sterilization of surgical materials and discuss their proper application in the hospital routine.

\section{RESULTS}

All patients underwent phacoemulsification with IOL implantation into the capsular bag. There were no complications during any of these surgeries. A sterile drape with an adhesive foil was used to remove the eyelashes from the operative field. Povidone-iodine was administered topically prior to surgery.

Prior to the endophthalmitis outbreak, the tubing kit of the phacoemulsification machine was used in 3 cataract surgeries per day. Only the 
phaco probe was exchanged between surgeries. Disinfection of the tubing kit was performed according to the manufacturer's recommendations (Allergan ${ }^{\circledR}$ ) and the material was autoclaved at the hospital's central sterilization facility.

A total of 140 patients were submitted to surgery in the same operating room between the first and last detected case of endophthalmitis, following the routine procedures described above. However, only 7 patients develop signs or symptoms suggestive of endophthalmitis.

All patients were initially treated with empiric administration of $0.4 \mathrm{mg}$ amikacin + $1 \mathrm{mg}$ vancomycin + $0.4 \mathrm{mg}$ dexamethasone (IV AVD; 0.1mL each) Vitreous samples were collected and plated on blood agar, chocolate agar, Sabouraud dextrose agar, thioglycollate broth and anaerobic medium. Gram was also performed. Antibiograms were performed in all cases for clinical guidance. Bacterial resistance was evaluated, but since $O$. anthropi has not been standardized by the Clinical Laboratory Standard Institute (CLSI) - the method used in our department of microbiology - the respective levels of sensitivity could not be determined.

Table 1 is a summary of patient data.

\section{Case 1}

A 73-year-old woman was submitted to surgery in the left eye (OS) on July 24. On the day after surgery, an anterior chamber inflammation was observed with flare (1+) and cells $(2-3+)$, in addition to a fibrinous pupillary membrane. Steroid therapy was started. On the following day, the ocular inflammation worsened. A clinical diagnosis of endophthalmitis was established and the patient was treated with IV AVD. O. anthropi was isolated in culture. The 
patient experienced visual improvement but white granular debris was noticed in the lower portion of the IOL.

On September 16, signs and symptoms of endophthalmitis were seen again and a new course of intravitreal antibiotics was initiated. One week later, a branch vein obstruction near the macula was observed.

Clinical follow-up showed complete reduction of intraocular inflammation, but on October 13 endophthalmitis recurred. On the following day, a pars plana vitrectomy (PPV) with partial capsulectomy (PC) plus meropenem injection and silicone oil was done. An area of necrosis in the periphery of the lower retina was observed and endolaser photocoagulation was performed to surround the lesion. This time there was no recurrence.

\section{Case 2}

A 59-year-old woman was submitted to surgery in the right eye (OD) on July 30. The patient complained of blurred vision on the tenth day after the surgery but only sought medical care 3 weeks later. She reported no pain during all this period. The examination showed a moderate vitritis with no anterior chamber activity. Due to only partial response to 3 weeks of steroid therapy, IV AVD was administered on September 22. O. anthropi was isolated in culture. No recurrence has been observed so far.

\section{Case 3}

A 79-year-old man was submitted to surgery in the OS on August 20. He began to complain of mild ocular pain and reduced visual acuity on the seventh day post-surgery (POD). The examination revealed corneal edema (3+), 
anterior chamber inflammation with flare (1+) and cells (2+), mild hypopyon and prominent vitritis. Clinical improvement was observed after treatment with IV AVD. O. anthropi was isolated in culture.

On October 18, the patient experienced another visual deterioration. On ophthalmoscopy, diffuse retinal hemorrhage was seen on the posterior pole, with moderate vitreous haze. No sign of endophthalmitis was observed this time.

\section{Case 4}

A 77-year-old woman was submitted to surgery in the OS on August 25. On POD 6, despite the absence of ocular pain, fibrin in the anterior chamber, cells $(2-3+)$, flare (1+) and mild hypopyon associated with the fibrinous pupillary membrane were observed (Figure 1). The patient was treated with IV AVD. $O$. anthropi was isolated in culture.

On September 29, the patient experienced a recurrence with anterior chamber inflammation (cells 3-4+), diffuse keratic precipitates, posterior synechiae and vitreous haze. A new course of amikacin and dexamethasone was initiated. Despite treatment, the patient developed dense and diffuse fibrin and hypopyon occupying one third of the anterior chamber. Antibiogram testing of the second sample indicated resistance to amikacin.

On October 20, PPV with PC plus injection of meropenem and silicone oil was performed. A large area of necrosis was observed in the lower retina and endolaser photocoagulation was used to surround the lesion. The inflammatory process persisted, with recurrence of hypopyon. On November 22, the patient was submitted to PPV with total capsulectomy, lensectomy and silicone oil 
removal and a new application of meropenem. The affected eye progressed to amaurosis and phthisis bulbi.

\section{Case 5}

A 76-year-old woman was submitted to surgery in the OD on September 1. On the fourth day after surgery the patient complained of mild ocular pain associated with decreased vision. Biomicroscopy showed anterior chamber inflammation with cells $(2+)$, flare (2-3+), linear hypopyon and fibrin at the pupillary edge. Given the uncertainty about etiology of the inflammation, steroid therapy was initiated. On the following day the hypopyon disappeared and visual acuity improved.

Five weeks after the surgery it was not yet possible to visualize the ocular fundus due to vitreous haze. On October 6, the patient started treatment with IV AVD, but no material was collected due to dry aspiration.

After a chronic intraocular inflammation with 3 episodes of exacerbation that partially responded to topical steroids, the patient was submitted to PPV on February 8 , including intravitreal application of amikacin and ceftazidime. During the surgery, pallor of the optic nerve was noticed. O. anthropi was isolated in culture.

The patient experienced good visual clinical improvement but died secondary to a proximal femoral fracture.

\section{Case 6}

A 60-year-old woman was submitted to surgery in the OS on September 27. On POD 3, the patient complained of ocular pain and decreased visual 
acuity. The examination revealed corneal edema (3+), anterior chamber inflammation with flare (1+) and cells (1+), fibrinous pupillary membrane and prominent vitritis. IV AVD was administered. No organism grew in the culture. Following the resolution of the inflammatory process on the 11th day, cottonwool spots, retinal hemorrhages and a macular hole were observed (Figure 2). The patient was subsequently lost to follow-up.

\section{Case 7}

A 71-year-old man was submitted to surgery in the OS on November 10. Six days later, severe eye pain and marked ocular inflammation with hypopyon occupying one third of the anterior chamber and fibrinous pupillary membrane was observed. The patient was treated with IV AVD. O. anthropi was isolated in culture. Considerable anterior chamber activity with no pain was still observed after three weeks. On ultrasound, small vacuoles were seen in the vitreous cavity. A new intravitreal injection of amikacin + dexamethasone was administered.

On December 18 the patient returned with severe ocular inflammation and pain again and was submitted to PPV with intravitreal meropenem and dexamethasone injection. During the surgical procedure, severe vitreous hemorrhage, many areas of retinal hemorrhage and macular fibrosis were observed. The patient was treated with panretinal endolaser photocoagulation and bevacizumab. A month later, endophthalmitis recurred and the patient was submitted to PPV with lensectomy and application of meropenem. The clinical follow-up revealed proliferative vitreoretinopathy followed later by vitreous 
hemorrhage. On June 3, the OS was eviscerated due to the persistence of the inflammatory process.

Since $O$. anthropi had not previously been isolated in our hospital, the protocols of infection control were instituted only when the Department of Ophthalmology became aware of a second case (case 4, September 2). The ophthalmic operating room was closed for 3 weeks while individual cases and the literature were reviewed.

We replaced several materials and supplies suspected of being the source of contamination, including IOL, mydriatic eyedrops, povidone-iodine solution and viscoelastic and balanced salt solution. Samples of these materials were submitted to laboratory analysis. In addition, all sterilization procedures and routines of the operating room were reviewed.

Safety precautions were taken and cataract surgeries restarted. However, six weeks later a new case of $O$. anthropi endophthalmitis (case 7) was identified. Another patient (case 6), submitted to surgery only four days after the operating room was reopened, was not diagnosed until the following year. The only point in common with the other surgeries was the tubing kit of the phacoemulsification machine. Flushings were then collected from four sets (all in use at the time) and submitted to culture along with the tubing. Fluid was collected both when flowing through the irrigation system and when returning from the aspiration system and drainage bag, pumping it both ways through the phaco probe.

While completing the purchase of new sets, we performed extracapsular cataract extraction with lens implantation without further cases of 
endophthalmitis. The situation was maintained after the resumption of phacoemulsification surgery.

All samples of materials sent to the microbiology laboratory had negative cultures. The Committee of Hospital Infection Control concluded that no specific cause could be identified, but contamination of the tubing kit is the most plausible explanation.

\section{DISCUSSION}

O. anthropi is a ubiquitous gram-negative, non-fastidious, highly motile, non-lactose fermenting bacillus with strictly oxidative metabolism and very active urease ${ }^{11-23}$ found mainly in water sources (including normal saline, antiseptic solutions and dialysis liquids). ${ }^{10-13}$ It is therefore likely to occupy the same microbial niche as Pseudomonas species. ${ }^{14}$ The average time required to grow of the cultures was 5 days in our cases (range: 4-6 days), which is consistent with previous reports. ${ }^{12}$

O. anthropi infrequently causes human infection, usually associated with immunocompromised hosts and indwelling catheters. ${ }^{13-21}$ This may be due to its ability to adhere to various synthetic materials such as IOL and silicone tubing (demonstrated by in vitro experiments). ${ }^{22}$ In 1980, the first case of human infection with $O$. anthropi was described in a patient with a pancreatic abscess. ${ }^{11}$ Since then, the pathogen has been described in six reported cases of endophthalmitis following intraocular surgery ${ }^{12,13,18,19,23}$ or by endogenous spread, ${ }^{20}$ with a total of 14 patients affected. 
The interval between cataract surgery and onset of signs and symptoms of endophthalmitis in this report ranged from 1-10 days (average: 5 days). This period was shorter than in previous studies (average: 6 weeks; range: 9-70 days). ${ }^{12,18,19,23}$

The clinical presenting signs were similar to $O$. anthropi pseudophakic endophthalmitis reported in the literature. The main presenting features were decreased vision (7/7), anterior chamber reaction (6/7), vitritis (7/7), hypopyon (5/7) and fibrinous pupillary membrane (4/7). However, the severity of disease found in this study contradicted the low virulence observed in previous reports. ${ }^{12,18,19,23}$ Final visual acuity was counting fingers or worse in $85 \%$ of patients (6/7) after the final clinical follow-up in this report, contrasting with the much better visual outcome observed in the literature $(20 / 60$ or better in 11 of 14 patients). Moreover, ocular complications occurred which had never been described in $O$. anthropi endophthalmitis, such as macular hole, venous occlusion, cotton-wool spots, retinal hemorrhage, necrosis and neovascularization. These findings may account for the poor visual acuity of our patients and suggest an underlying vasculitis or perivasculitis.

Several studies have shown that microorganisms can be isolated from the anterior chamber at the end of cataract surgery in 5 to $43 \%$ of patients. ${ }^{24-28}$ This fact does not necessarily imply infection, suggesting that natural defense mechanisms are capable of eliminating small inocula of bacteria of low pathogenicity thereby preventing the development of endophthalmitis. ${ }^{7}$ This appears to be the case of $O$. anthropi, which, despite the severity of our cases, presented an attack rate of approximately $5 \%$ during the period of the outbreak, 
considering individuals at submitted to phacoemulsification in any intraocular surgery.

All patients had vision of hand motions or better at the initial presentation. Following the guidelines of the Endophthalmitis Vitrectomy Study Group, ${ }^{29-33}$ patients initially received empiric treatment with intravitreal antibiotics plus dexamethasone, and vitreous samples were collected for microbiological investigation. The use of aminoglycosides in our hospital is due to its bactericidal dose-dependent action (even at high concentrations of germs) and synergism with vancomycin - characteristics not observed with ceftazidime. ${ }^{34}$

The use of intravitreal corticosteroids in the management of endophthalmitis remains controversial. ${ }^{35}$ However, several clinical and experimental reports suggest that intravitreal corticosteroid therapy used in conjunction with antibiotics may reduce the intraocular inflammatory process and secondary complications associated with microbial endophthalmitis. ${ }^{34-36}$

In this report, initial therapy with intravitreal injection of antibiotics proved ineffective in 5 of 7 cases (71\%). PPV combined with PC, or even total capsulectomy and IOL removal, as described in the literature, does seem to be curative. $^{12,19,23}$ Innate resistance to antibiotics ${ }^{11-23}$ and sequestration in the capsular bag ${ }^{12,19,23}$ justify the need for these additional procedures. A factor probably responsible for this situation is the formation of a biofilm, ${ }^{37,38}$ a functional consortium of micro-organisms organized within an extensive exopolymer matrix ${ }^{39}$ which confers relative protection from humoral and cellular immunity and from antibiotics. ${ }^{3}$ In addition, Griffiths et $a l^{40}$ found that adherence of Staphylococcus epidermidis to IOLs in vitro appears to confer greater 
resistance to antibiotics, while Cusumano et $a^{41}$ demonstrated that bacterial growth in vitro is significantly enhanced on silicone IOLs.

Perhaps in an attempt to reduce costs and increase the efficiency of the phacoemulsification machine, it is common practice to use a single tubing kit to perform multiple cataract surgeries on the same day, exchanging only the phaco probe (or simply the phaco tip) between surgeries. However, silicone material has been shown to allow adhesion of bacteria and other biomaterials ${ }^{22,41,42}$ and in some studies standard cleaning regimes were shown not to be completely effective at removing debris from previous surgeries. ${ }^{4,43}$ Various contaminants were identified, including lens capsule and cells, manmade fibers, squamous cells, bacteria, fungal elements, diatoms, red blood cells and proteinaceous material. ${ }^{4}$ The presence of organic debris on instruments interferes with the sterilization process as it insulates the organisms from the killing agent. This fact favors the formation of bacterial biofilm and puts patients at risk for cross-infection. ${ }^{44,45}$ This was probably the source of our outbreak of endophthalmitis.

Due to the lack of an established cleaning and sterilization protocol in the literature, ${ }^{4,5,43,46,47}$ a new protocol was designed at our hospital: the tubing is flushed with distilled water through the purge cycle of the phacoemulsification machine (repeated three times) immediately after each surgery, according to the manufacturer's recommendations. Upon arrival at the central sterilization facility, the tubing is placed in an ultrasonic bath with a solution of enzymatic detergent $\left(\right.$ Maxzyme $\left.^{\circledR}\right)$ at $50{ }^{\circ} \mathrm{C}$ for 10 minutes to remove the debris before sterilization. The tubing is subsequently washed with water, cleaned with compressed air and placed in the autoclave. All tubing kits are submitted to 
sterility assurance level testing and are sent to the microbiology laboratory to search for microbial contamination after being used twenty times.

Intraocular surgery using the phacoemulsification machine was recommenced following the implementation of the above sterilization protocol. At the time of writing, 1008 cataract surgeries have been performed without a single case of postoperative endophthalmitis and no further contamination of the tubing kits.

It is also worthy of mention that following the implementation of the new protocol, no cases of Toxic Anterior Segment Syndrome ${ }^{48,49}$ have been observed at our hospital despite the use of materials and procedures known to be implicated with this syndrome, such as enzymatic detergents and ultrasonic cleaning.

\section{CONCLUSION}

This report describes a series of cases of $O$. anthropi endophthalmitis with unusually severe outcomes, including several complications never reported in the literature. Inefficient sterilization can be a source of endophthalmitis

outbreaks. However, it seems cross-infection can be prevented by vigorous cleaning of the tubing with distilled water and ultrasonic cleaning using an enzymatic detergent to remove the debris before sterilization to prevent the formation of bacterial biofilm. Cataract surgery represents a substantial cost to health care systems around the world. The new sterilization protocol proposed in this paper offers improved safety when reusing tubing in phacoemulsification. 


\section{WHAT WAS KNOWN}

* Only 14 cases of Ochrobactrum anthropi endophthalmitis were reported in the literature at the time of writing. Usually, this bacteria cause subacute or chronic disease that evaluated with a good visual outcome.

* Silicone material has been shown to allow adhesion of bacteria and other biomaterials and in some studies standard cleaning regimes were shown not to be completely effective at removing debris from previous surgeries.

\section{WHAT THIS PAPER ADDS}

* Our observations differ from previously reported cases of $O$. anthropi pseudophakic endophthalmitis due to the shorter time of onset of signs and symptoms, the severity of the disease and complications not previously reported.

* Cataract surgery represents a substantial cost to health care systems around the world. Persistence in the practice of reusing tubing is controversial and requires risk-benefit analysis at every hospital or clinic that performs cataract surgery. The new sterilization protocol proposed in this paper offers improved safety when reusing tubing in phacoemulsification. 


\section{REFERENCES}

1- Novosad BD. Callegan MC. Severe bacterial endophthalmitis: towards improving clinical outcomes. Expert Rev Ophthalmol 2010;5(5):689-698.

2- Li J, Morlet N, Ng JQ, Semmens JB, Knuiman MW. Significant nonsurgical risk factors for endophthalmitis after cataract surgery: EPSWA Fourth Report. Invest Ophthalmol Vis Sci 2004;45(5):1321-8.

3- Bainbridge JWB, Teimory M, Tabandeh H, Kirwan JF, Dalton R, Reid F, Rostron CK. Intraocular lens implants and risk of endophthalmitis. $\mathrm{Br} \mathrm{J}$ Ophthalmol 1998;82:1312-5.

4- Leslie T, Aitken DA, Barrie T, Kirkness CM. Residual debris as a potential cause of postphacoemulsification endophthalmitis. Eye 2003;17:506-512.

5- Anderson OA, Lee V, Shafi S, Keegan D, Vafidis G. A model for the management of an atypical endophthalmitis outbreak. Eye 2005;19:972-980.

6- Miller JJ, Scott IU, Flynn HWJ, Smiddy WE, Newton J, Miller D. Acute-onset endophthalmitis after cataract surgery (2000-2004): incidence, clinical settings, and visual acuity outcomes after treatment. Am J Ophthalmol 2005;139(6):9837.

7- Callegan MC, Engelbert M, Parke DW 2nd, Jett BD, Gilmore MS. Bacterial endophthalmitis: epidemiology, therapeutics, and bacterium-host interactions. Clin Microbiol Rev 2002;15(1):111-24.

8 - Mayer E, Cadman D, Ewings P, Twomey JM, Gray RH, Claridge KG, Hakin $\mathrm{KN}$, Bates AK. A 10 year retrospective survey of cataract surgery and endophthalmitis in a single eye unit: injectable lenses lower the incidence of endophthalmitis. Br J Ophthalmol 2003;87:867-9. 
9- Rogers NK, Fox PD, Noble BA, Kerr K, Inglis T. Aggressive management of an epidemic of chronic pseudophakic endophthalmitis: results and literature survey. Br J Ophthalmol 1994;78:115-119.

10- Saraiva FP, Costa PG, Inomata DL, Preti RC, Helal Júnior J, Nakashima Y. Clinical profile of patients with endophthalmitis hospitalized at Hospital das Clínicas of São Paulo. Rev Bras Oftalmol 2007;66(3):169-74.

11- Appelbaum PC, Campbell DB. Pancreatic abscess associated with Achromobacter group Vd biovar 1. J Clin Microbiol 1980;12:282-3.

12- Song S, Ahn JK, Lee GH, Park YG. An epidemic of chronic pseudophakic endophthalmitis due to Ochrobactrum anthropi: clinical findings and managements of nine consecutive cases. Ocul Immunol Inflamm 2007;15(6):429-34.

13- Inoue K, Numaga J, Nagata Y, Sakurai M, Aso N, Fujino Y. Ochrobactrum anthropi endophthalmitis after vitreous surgery. $\mathrm{Br} \quad \mathrm{J}$ Ophthalmol 1999;83(4):502.

14- Kettaneh A, Weill FX, Poilane I, Fain O, Thomas M, Herrmann JL, Hocqueloux L. Septic shock caused by Ochrobactrum anthropi in an otherwise healthy host. J Clin Microbiol 2003;41(3):1339-41.

15- Cieslak T J, Robb ML, Drabick CJ, Fischer GW. Catheter associated sepsis caused by Ochrobactrum anthropi: report of a case and review of related nonfermentative bacteria. Clin Infect Dis 1992;14:902-7.

16- Gómez MPR, Esteban AMP, Daza JAS, Nieto JAS, Alvarez D, García PP. Prosthetic Mitral Valve Endocarditis Due to Ochrobactrum anthropi: case report. J Clin Microbiol 2004;42(7):3371-3. 
17- Vaidya SA, Citron DM, Fine MB, Murakami G, Goldstein EJC. Pelvic abscess due to Ochrobactrum anthropi in an immunocompetent host: case report and review of the literature. J Clin Microbiol 2006;44(3):1184-6.

18- Greven CM, Nelson KC. Chronic postoperative endophthalmitis secondary to Ochrobactrum anthropi. Retina $2001 ; 21(3): 279-80$.

19 -Chiang CC, Tsai YY, Lin JM, Chen WL. Chronic endophthalmitis after cataract surgery secondary to Ochrobactrum anthropi. Eye 2009;23(5):1237-8.

20- Berman AJ, Del Priore LV, Fischer CK. Endogenous Ochrobactrum anthropi endophthalmitis. Am J Ophthalmol 1997;123(4):560-2.

21- Deliere E, Vu-Thien H, Levy V, Barquins S, Schlegel L, Bouvet A. Epidemiological investigation of Ochrobactrum anthropi strains isolated from a haematology unit. J Hosp Infect 2000;44:173-8.

22- Wi YM, Sohn KM, Rhee JY, Oh WS, Peck KR, Lee NY, Song JH. Spontaneous bacterial peritonitis due to Ochrobactrum anthropi: a case report. J Korean Med Sci 2007;22:377-9.

23- Braun M, Jonas JB, Schönherr U, Naumann GO. Ochrobactrum anthropi endophthalmitis after uncomplicated cataract surgery. Am $\mathrm{J}$ Ophthalmol 1996;122(2):272-3.

24- Durand ML. Bacterial endophthalmitis. Curr Infect Dis Rep 2009;11(4):2838.

25- Beigi, B., W. Westlake, E. Mangelschots, B. Chang, W. Rich, and T. Riordan. Peroperative microbial contamination of anterior chamber aspirates during extracapsular cataract extraction and phacoemulsification. Br. J. Ophthalmol 1997;81:953-955. 
26- Arantes TEF, Castro CMMB, Cavalcanti RF, Severo MS, Diniz MFA, Urtiga RWD. Conjunctival bacterial flora after topical use of ciprofloxacin and gatifloxacin in cataract surgery. Arq Bras Oftalmol 2008;71(2):191-6.

27- Dickey JB, Thompson KD, Jay WM. Anterior chamber aspirate cultures after uncomplicated cataract surgery. Am J Ophthalmol 1991;112(3):278-82. Comment in: Am J Ophthalmol 1992;113(2):221-2.

28- Samad A, Solomon LD, Miller MA, Mendelson J. Anterior chamber contamination after uncomplicated phacoemulsification and intraocular lens implantation. Am J Ophthalmol 1995;120(2):143-50.

29- Endophthalmitis Vitrectomy Study Group. Results of the Endophthalmitis Vitrectomy Study: a randomized trial of immediate vitrectomy and of intravenous antibiotics for the treatment of postoperative bacterial endophthalmitis. Arch Ophthalmol 1995;113(12):1479-1496.

30- Endophthalmitis Vitrectomy Study Group. Microbiologic factors and visual outcome in the Endophthalmitis Vitrectomy Study. Am J Ophthalmol 1996;122(6):830-846.

31- Smiddy WE, Smiddy RJ, Ba'Arath B, et al. Subconjunctival antibiotics in the treatment of endophthalmitis managed without vitrectomy. Retina $2005 ; 25(6): 751-758$.

32- Iyer MN, Han DP, Yun HJ, et al. Subconjunctival antibiotics for acute postcataract extraction endophthalmitis: is it necessary? Am J Ophthalmol 2004;137(6):1120-1121.

33- Flynn HWJ, Scott IU. Legacy of the endophthalmitis vitrectomy study. Arch Ophthalmol 2008;126(4):559-61. 
34- Serracarbassa PD. Serracarbassa LL. Rodrigues LD. Antibióticos intravítreos. Arq Bras Oftalmol 2003;66:527-30.

35- Das T, Jalali S, Gothwal VK, Sharma S, Naduvilath TJ. Intravitreal dexamethasone in exogenous bacterial endophthalmitis: results of a prospective randomised study. Br J Ophthalmol 1999;83(9):1050-5.

36- Schulman JÁ, Peyman GA. Intravitreal corticosteroids as an adjunct in treatment of bacterial and fungal endophthalmitis: a review. Retina 1992;12:336-40.

37- Cusumano A, Busin M, Spitznas M. Is chronic intraocular inflammation after lens implantation of bacterial origin? Ophthalmology 1991;98(11):1703-10.

38- Morlet $\mathrm{N}$, Li J, Semmens $\mathrm{J}, \mathrm{Ng} \mathrm{J}$, on behalf of team EPSWA. The Endophthalmitis Population Study of Western Australia (EPSWA): first report. $\mathrm{Br}$ J Ophthalmol 2003;87:574-576.

39- Costerton JW, Cheng KJ, Geesy GG, Ladd TI, Nickel JC, Dasgupta M, Marrie TJ. Bacterial biofilms in nature and disease. Annu Rev Microbiol $1987 ; 41: 435-64$.

40- Griffiths PG, Elliott TSJ, McTaggart L. Adherence of Staphylococcus epidermidis to intraocular lenses. $\mathrm{Br} \mathrm{J}$ Ophthalmol 1989;73(6):402-6.

41- Cusumano A, Busin M, Spitznas M. Bacterial growth is significantly enhanced on foldable intraocular lenses. Arch Ophthalmol 1994;112(8):101516.

42- Gabriel MM, Ahearn DG, Chan KY, Patel AS. In vitro adherence of Pseudomonas aeruginosa to four intraocular lenses. J Cataract Refract Surg 1998;24:124-129. 
43- Dinakaran S, Kayarkar VV. Debris on processed ophthalmic instruments: a cause for concern. Eye 2002;16:281-284.

44- Miller $\mathrm{CH}$. Cleaning, sterilization and disinfection: basics of microbial killing for infection control. J Am Dent Assoc 1993;124:48-56.

45- Garcia TV. Estudo experimental sobre a contaminação por vírus no instrumental cirúrgico da facoemulsificação. 2009. 74 f. Tese (Mestrado em Ciências Médicas) - Faculdade de Medicina de Ribeirão Preto, Universidade de São Paulo, Ribeirão Preto. 2009.

46- Marion-Ferey K, Pasmore M, Stoodley P, Wilson S, Husson GP, Costerton JW. Biofilm removal from silicone tubing: an assessment of the efficacy of dialysis machine decontamination procedures using an in vitro model. J Hosp Infect 2003;53(1):64-71.

47- Rutala WA, Weber DJ and the Healthcare Infection Control Practices Advisory Committee (HICPAC). CDC - Guideline for Disinfection and Sterilization in Healthcare Facilities, 2008.

48- Cutler Peck CM, Brubaker J, Clouser S, Danford C, Edelhauser HE, Mamalis N. Toxic anterior segment syndrome: common causes. J Cataract Refract Surg. 2010;36(7):1073-80.

49- van Philips LA. Toxic anterior segment syndrome after foldable artiflex irisfixated phakic intraocular lens implantation. J Ophthalmol. 2011;2011:982410. Epub 2011 Jun 5. 
FIGURE LEGEND

FIGURE 1: Visualization of pupillary membrane and small hypopyon on slit lamp examination of case 4 .

FIGURE 2: Cotton-wool spots, retinal hemorrhages and a macular hole are observed on fundus examination of case 6 . 